\title{
Risk Acceptance and Expectations of Laryngeal Allotransplantation
}

\author{
Hyun Kyo Jo ${ }^{1}$, Jang Wan Park ${ }^{1}$, Jae Ha Hwang ${ }^{1}$, Kwang Seog Kim , Sam Yong Lee ${ }^{1}$, Jun Ho Shin ${ }^{2}$ \\ Departments of ${ }^{1}$ Plastic and Reconstructive Surgery and ${ }^{2}$ Preventive Medicine, Chonnam National University Medical School, Gwangju, Korea
}

Background Laryngeal allotransplantation (LA) is a technique involving transplantation of a deceased donor's larynx into a recipient, and it may be substituted for conventional laryngeal reconstruction. There are widely different views on $L A$, as the recipient is administered continuous, potentially life-threatening, immunosuppressive therapy for a functional or aesthetic result, which is not directly related to life extension. The purpose of this study was to analyze the difference in risk acceptance and expectations of LA between four population groups.

Methods A survey was performed to examine patients' risk acceptance and expectations of LA. The survey included 287 subjects in total (general public, $n=100 ;$ kidney transplant recipients, $n=53$; post-laryngectomy patients, $n=34$; doctors, $n=100$ ), using a Korean translated version of the louisville instrument for transplantation (LIF) questionnaire.

Results All four groups responded differently at various levels of their perception in risk acceptance and expectations. The kidney transplant recipients reported the highest risk acceptance and expectations, and the doctor group the lowest.

Conclusions This study examined the disparate perception between specific population groups of the risks and benefits of using LA for the promotion of the quality of life. By addressing the information gaps about LA in the different populations that have been highlighted from this survey, we suggest that LA can become a more viable alternative to classical surgery with resultant improved quality of life for patients.

Keywords Larynx / Reconstructive surgical procedures / Patient acceptance of healthcare / Data collection
Correspondence: Kwang Seog Kim Department of Plastic and Reconstructive Surgery, Chonnam National University Medical School, 42 Jebong-ro, Dong-gu, Gwangju 501-757, Korea Tel: +82-62-220-6363/6352 Fax: +82-62-227-1639 E-mail: pskim@chonnam.ac.kr

No potential conflict of interest relevant to this article was reported.

Received: 26 Mar 2014 • Revised: 9 Jun 2014 • Accepted: 14 Jun 2014

pISSN: 2234-6163 • elSSN: 2234-6171 • http://dx.doi.org/10.5999/aps.2014.41.5.505 • Arch Plast Surg 2014;41:505-512

\section{INTRODUCTION}

Quality of life is important in patients who need removal of a damaged larynx because of severe trauma or head and neck cancer. With laryngectomy, the patient can no longer speak normally, and depends on an assistive device, which is limited by number and practicality, or esophageal speech, which is limited by practicality. McNeil et al. [1] showed that $20 \%$ of their study participants preferred to maintain vocal function by using radiotherapy to treat their cancer, even though radiation therapy offers only a $30 \%$ to $40 \%$ 3-year survival rate, while laryngectomy provides a $60 \%$ 3-year survival rate.

Laryngeal allotransplantation (LA) is a procedure whereby the larynx of a deceased donor is transplanted into a recipient. Classical laryngeal reconstruction, such as flap surgery, skin graft, or implantation of artificial material, has limited indica- 
tions, and therefore, LA may offer an alternative approach. The procedure has been performed in animals, especially in dogs by Work and Boles [2], Ogura et al. [3], and Silver et al. [4]. Since Kluyskens and Ringoir [5] in 1969 first tried it in humans, it has been used on patients with trauma or tumors. In 1988, Strome et al. [6] and in 2007, Tintinago et al. [7] and Duque et al. [8] obtained organ functionality and satisfactory aesthetic results from transplanting composite tissues, including the larynx and the tracheopharyngeal complex. However, in both studies, use of LA by the investigators was limited by immunosuppression and tissue rejection [9-12].

Unlike liver and kidney allotransplantations, which are currently being used, the LA procedure is often criticized because allotransplantation requires administration of continuous, lifethreatening immunosuppression therapy for a functional or aesthetic outcome with no impact on survival [13]. Moreover, patients may have ethical objections or experience psychological stress when undergoing LA as the larynx is collected from a deceased donor [14].

Several studies in other countries have investigated patients' risk acceptance and expectations of LA with composite tissue allotransplantation [15-18]. Similarly, in Korea, studies have investigated patients' risk acceptance and expectations of allotransplantation operations on their face, hands, and feet $[19,20]$. However, insufficient research into LA has been performed in Korea, and the larynx has its own distinct features and characteristics that contrast with other composite tissues. Therefore, using similar survey methods to previous studies, we analyzed the differences between specific population groups in their perception and risk acceptance and expectations of LA.

\section{METHODS}

\section{Samples}

The survey to find out patients' risk acceptance and expectations of LA was conducted by dividing subjects into four groups: the first group of members of the general public who had little or no expert knowledge, the second group of kidney transplant recipients who had experienced immunosuppressive therapy directly, the third group of patients who are targeted at LA and the fourth group of doctors who had experience of performing medical procedures, medical knowledge, and clinical experience.

\section{General public group}

We surveyed 100 guardians of outpatients and inpatients at Chonnam National University Hospital. Study participants were between 24 and 71 years old (average age, 43.23 years; standard deviation $[\mathrm{SD}], 12.77$ ) and were surveyed from March
2012 to April 2014.

\section{Kidney transplant recipients group}

During the same time of period, kidney transplant recipients were surveyed at Chonnam National University Hospital. The recipients were around 26 to 64 years old (average age, 46.69 years; SD, 9.71).

\section{Post-laryngectomy patients group}

We surveyed 34 post-laryngectomy patients who had operations at Chonnam National University Hospital, whose age group from 38 to 75 years old (average age, 59.85 years; SD, 9.52).

\section{Doctor group}

We surveyed 100 doctors from 27 to 37 years old (average age, 30.09 years; SD, 2.71) working at Chonnam National University Hospital.

\section{METHODS}

\section{Study design}

Brouha et al. [16] invented the louisville instrument for transplantation (LIFT), which contains a questionnaire that sets virtual scenarios to evaluate the amount of risk individuals can endure balanced with the benefit of composite tissue allotransplantation. The virtual clinical scenarios presented described seven different organ and tissue transplants, including foot, kidney, hand, both hands, larynx, part of the face, and full face. In addition, the questionnaire records the respondents' thoughts on various subjects, self-evaluation, and personal information related to ethnicity, religion, occupation, income, and education level.

Kim et al. [21], Park et al. [19], and Lee et al. [20] translated the LIFT questionnaire into a Korean language version and carried out surveys (which is recognized in its validity and reliability around English culture) to assess patients' risk acceptance and expectations for face, hand, or foot allotransplantation. Similarly, in this study, the Korean version of the LIFT, particularly an extract from a part specifically about LA, was used for the survey. Kim et al. [21] had proved that the Korean version of the LIFT is a useful tool for the clinic; thus, this study provided valid information of Koreans' perception of risk acceptance and expectations of LA. The survey questionnaire was filled out in person and any parts that were difficult to understand or missing were completed through private interviews with the participants.

Prior to completing the questionnaire, all subjects were provided with further details of composite tissue allotransplantation, and the ethical and social controversies around the LA 
procedure. To aid in their understanding, the survey participants viewed preoperative and postoperative pictures and a postoperative video recording of vocalization by a patient who underwent laryngectomy. Moreover, subjects were provided with an explanation of the side effects of immunosuppression from taking antirejection medications postoperatively. Less serious complications included relatively mild symptoms of diarrhea, constipation, nausea, vomiting, weight gain, and dizziness. Other more serious complications included urinary tract infection, hypertension, diabetes, kidney failure, headache, liver toxicity, atherosclerosis, osteoporosis, tumors of the lymph glands, skin, or major organs, and bacterial or viral infections. In addition, they were informed that life-threatening complications can occur while taking antirejection medications, which will need surgery.

\section{Survey/Statistical procedures}

Risk acceptance of laryngeal allotransplantation

The risk acceptance indicates the degree of harm or loss in order to obtain a certain gain. In this study, the side effects of immunosuppression and the risk of tissue rejection were separately examined.

\section{Risk acceptance of the side effects of immunosuppression}

We analyzed the "Yes" or "No" response of the willing participants to undergo the LA procedure despite the possible risk of immunosuppressive side-effects and also for the amount they had to give up in life due to the side effects of immunosuppression if they had only 10 years of remaining life (Fig. 1).

\section{Risk acceptance of tissue rejection}

The intention to undergo allotransplantation was assessed with the "Yes" or "No" answer when assuming a probability of a negative reaction in the first year after LA. After LA, the willingness to risk undergoing surgery when there was a possibility of tissue rejection was assessed (figures were expressed as percentages, and the higher the probability, the stronger the risk) (Fig. 2).

\section{Fig. 1. Questionnaire focused on risk acceptance of immunosuppression}

(A) Question inquiring about acceptance of immunosuppression side effects. (B) Question inquiring about maximum immunosuppression risk acceptability.

\section{Please read the below "patient informed consent" paragraph.}

After your transplant you will need to take antirejection medications for the rest of your life. The risks of these medications include: nausea, vomiting, diarrhea, constipation, weight gain, dizziness, urinary tract infections, hypertension, diabetes, kidney failure, headaches, liver toxicity; tumors of lymph glands, skin or major organs; atherosclerosis, osteoporosis, bacterial or viral infections. Even taking your medication, your body may still reject the transplant and it will need to be surgically removed. If any life threatening complications arise from the antirejection medications, these medications will need to be discontinued and the transplant will need to be surgically removed.

After reading the above would you still want to get a larynx transplant?

$$
\begin{array}{|l|l|}
\hline \square & \text { No } \\
\square & \text { Yes }
\end{array}
$$

Suppose you have 10 years to live. What is the maximum percent of remaining years you would give up to get a larynx transplant?

Maximum percent of remainingyears you would give up to get transplant (circle one)

\begin{tabular}{|l|l|l|l|l|l|l|l|l|l|l|l|l|} 
Give up NO & $0 \%$ & $10 \%$ & $20 \%$ & $30 \%$ & $40 \%$ & $50 \%$ & $60 \%$ & $70 \%$ & $80 \%$ & $90 \%$ & $100 \%$ & Give up ALL \\
\hline
\end{tabular}

\begin{tabular}{l|l|l|l|l|l|l|l|l|l|l|l|l|l|}
\hline \% of years & $\square$ & $\square$ & $\square$ & $\square$ & $\square$ & $\square$ & $\square$ & $\square$ & $\square$ & $\square$ & $\square$ & $\%$ of years \\
\hline
\end{tabular}

\section{Fig. 2. Questionnaire focused on risk acceptance of rejection}

(A) Question inquiring about acceptance of $50 \%$ chance of tissue rejection. (B) Question inquiring about maximum rejection risk acceptability.

If the chance of rejecting the larynx were $50 \%$ in the first year, would you still get the larynx transplant?

$\square \quad$ No

$\square \quad$ Yes

In all transplants there is always a chance of rejection, which could cause the complete loss of the

transplanted larynx. What is the maximum chance of rejection you would accept and still get the larynx transplant?

Chance of rejection (circle one)

\begin{tabular}{|l|l|l|l|l|l|l|l|l|l|l|l|l|}
\hline LOW & $0 \%$ & $10 \%$ & $20 \%$ & $30 \%$ & $40 \%$ & $50 \%$ & $60 \%$ & $70 \%$ & $80 \%$ & $90 \%$ & $100 \%$ & HIGH \\
chance of & & $40 \%$ of & \\
\hline
\end{tabular}

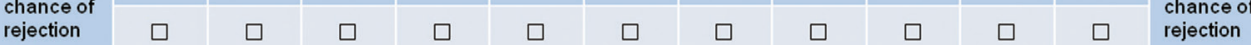




\section{Fig. 3. Questionnaire focused on quality of life}

(A) Question inquiring about quality of life if the larynx is destroyed.

(B) Question inquiring about expected quality of life after transplantation.

\section{- Virtual scenario -}

You sustained an injury that destroyed your larynx (voice box), the part of your throat that controls much of the ability to speak. You have some speech through an electronic device, but it is difficult to use, you must speak very slowly, and even then your voice sounds fla t and electronic, like a robot. People have difficulty understanding you.

If you lost your larynx, what do you think your quality of life would be (include your personal, social and occupational quality)?

\section{Quality of life (circle one)}

\begin{tabular}{|l|l|l|l|l|l|l|l|l|l|l|l|l|}
\hline $\begin{array}{l}\text { WORST } \\
\text { quality }\end{array}$ & 0 & 1 & 2 & 3 & 4 & 5 & 6 & 7 & 8 & 9 & 10 & BEST \\
\hline & $\square$ & $\square$ & $\square$ & $\square$ & $\square$ & $\square$ & $\square$ & $\square$ & $\square$ & $\square$ & $\square$ & quality \\
\hline
\end{tabular}

If you got the larynx transplant, what do you think your quality of life would be (include your personal, social and occupational quality)?

\section{Quality of life (circle one)}

\begin{tabular}{|l|l|l|l|l|l|l|l|l|l|l|l|l|l|}
\hline $\begin{array}{l}\text { WORST } \\
\text { quality }\end{array}$ & 0 & 1 & 2 & 3 & 4 & 5 & 6 & 7 & 8 & 9 & 10 & BEST \\
\cline { 1 - 8 } & $\square$ & $\square$ & $\square$ & $\square$ & $\square$ & $\square$ & $\square$ & $\square$ & $\square$ & $\square$ & $\square$ & quality & B \\
\hline
\end{tabular}

\section{Fig. 4. Questionnaire focused on expectations of functional outcomes}

\section{(A) Question inquiring} about expected function after transplantation. (B) Question inquiring about importance of function.
Suppose the antirejection medications would reduce your life span by ONE THIRD. Choose the minimum level of improvement the larynx transplant would have to provide for you to accept it? (100\% means maximal improvement (identical to normal) and $0 \%$ means no improvement).

\begin{tabular}{|l|c|c|c|c|c|c|c|c|c|c|c|c|c|}
\hline \multicolumn{10}{|c|}{ Minimum level of improvement(circle one) } \\
\hline $\begin{array}{l}\text { Minimal } \\
\text { improvement }\end{array}$ & $0 \%$ & $10 \%$ & $20 \%$ & $30 \%$ & $40 \%$ & $50 \%$ & $60 \%$ & $70 \%$ & $80 \%$ & $90 \%$ & $100 \%$ & $\begin{array}{l}\text { Maximal } \\
\text { improvement }\end{array}$ \\
\hline & $\square$ & $\square$ & $\square$ & $\square$ & $\square$ & $\square$ & $\square$ & $\square$ & $\square$ & $\square$ & $\square$ \\
\hline
\end{tabular}

In considering this transplant, how important is larynx function (return of voice) as opposed to appearance?

Importance of function (circle one)

\begin{tabular}{|l|c|c|c|c|c|c|c|c|c|c|c|c|}
\hline $\begin{array}{l}\text { Not } \\
\text { important }\end{array}$ & 0 & 1 & 2 & 3 & 4 & 5 & 6 & 7 & 8 & 9 & 10 & Extremely \\
important
\end{tabular}

Expectations of laryngeal allotransplantation

Expectations of quality of life

We surveyed the change expected in the quality of life after LA if the participants had a defect in their larynx or soon were expected to have a defect after surgery (the figures were represented as a number from 0 to 10 , with higher numbers mean higher expectations) (Fig. 3).

Function improvement after transplantation and importance of function and appearance

Assuming the antirejection medications after LA would reduce patients' life span by one third, each group had to answer about to what extent a reduced lifetime is acceptable to them when certain level of functional improvement occurs (displayed as a percentage from $0 \%$ to $100 \%$ and the higher probability means maximal improvement). Moreover, the subjects marked about how significant they consider the function of the transplanted larynx (restoration of voice) and appearance (image of being complete) into a number from 0 to 10 and the higher number means greater importance (Figs. 4, 5).

\section{Statistical analyses}

The data collected through the survey was organized using Microsoft Office Excel 2010 software (Microsoft Corp., Redmond, WA, USA) and was analyzed with PASW statistics ver. 18 (SPSS Inc., Chicago, IL, USA). One-way analysis of variance was used 


\section{Fig. 5. Questionnaire focused on expectations of aesthetic outcomes}

Question inquiring about importance of appearance.
In considering this transplant, how important is the appearance of having a normal voice (image of being complete) as opposed to function?

Importance of appearance (circle one)

\begin{tabular}{|l|c|c|c|c|c|c|c|c|c|c|c|c|c|}
\multicolumn{10}{c|}{} \\
\hline $\begin{array}{l}\text { Not } \\
\text { important }\end{array}$ & 0 & 1 & 2 & 3 & 4 & 5 & 6 & 7 & 8 & 9 & 10 & Extremely \\
important
\end{tabular}

Fig. 6. Risk acceptance in laryngeal allotransplantation

The ability to accept risk (general public, kidney transplant recipients, post-laryngectomy patients, and doctors) of immunosuppression and tissue rejection in laryngeal allotransplantation.

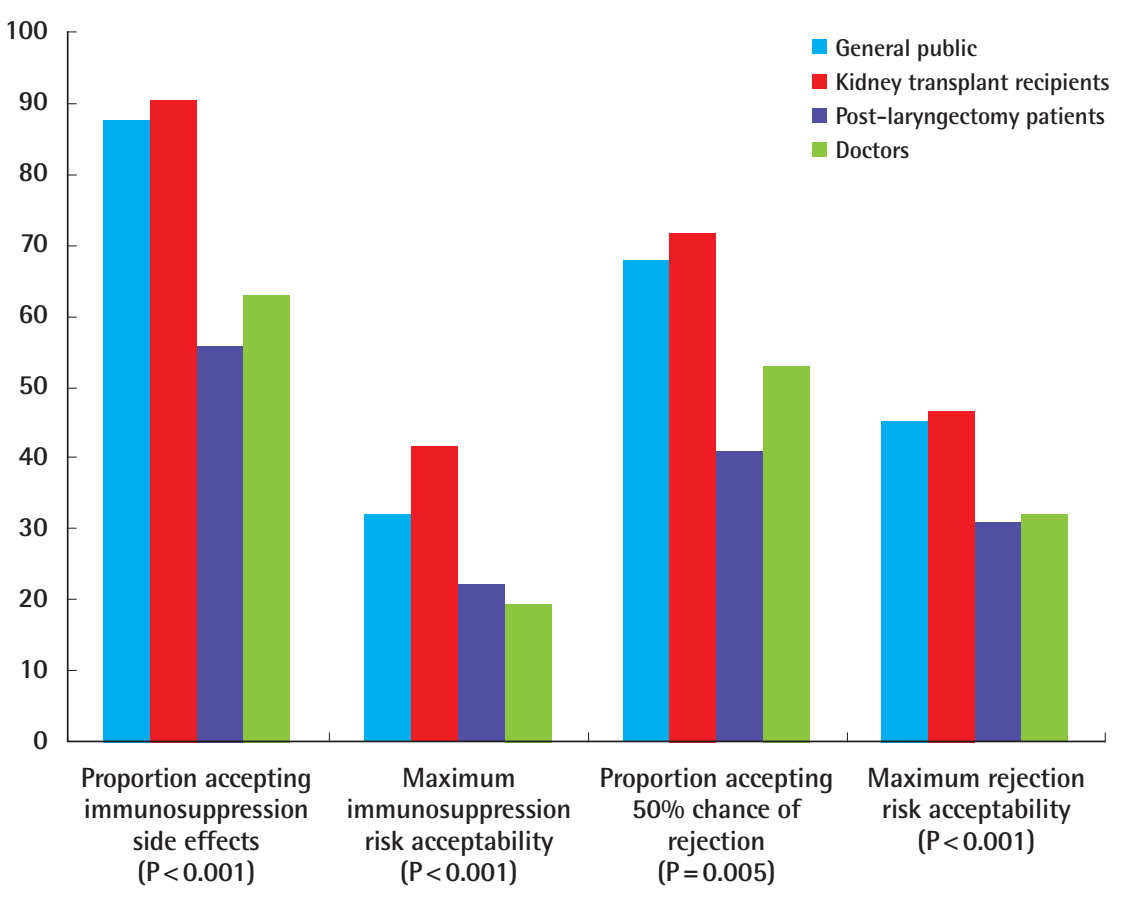

to compare the differences between groups and Duncan's posthoc multiple comparison was used to verify the further specific differences. In addition, the dichotomy "Yes" or "No" research questions concerning the risk acceptance were analyzed using the Chi square test with the significance level of less than 0.05 .

\section{RESULTS}

\section{Examination of risk acceptance of laryngeal allotransplantation}

Risk acceptance of the side effects of immunosuppression

When considering the side effects of immunosuppression, $88 \%$ of the general public group and $90.6 \%$ of the kidney transplant recipients group responded positively about LA. This was a higher level than both the post-laryngectomy patients group (55.9\%) and doctor group (63\%) $(\mathrm{P}<0.001)$. We asked the groups what was the maximum percentage of years that you would give up for larynx transplantation if you had 10 years to live, the average values in percentages from the subjects who responded were obtained. The results were as followings: the general public group, 31.9\%; kidney transplant recipients group, $41.7 \%$; post-laryngectomy patients group, $22 \%$; and doctor group, $19.4 \%$. The distinctions between the four groups were considerable according to one-way analysis of variance $(\mathrm{P}<$ 0.001 ), but there was no significant difference between the postlaryngectomy patients and doctor group according to post-hoc multiple comparison $(\mathrm{P}=0.428)$ (Fig. 6).

\section{Risk acceptance of tissue rejection}

When supposing a probability of rejection in the first year after LA of $50 \%, 68 \%$ of the general public group and $71.7 \%$ of the kidney transplant recipients group were interested in undergoing allotransplantation surgery. It was higher than the post-laryngectomy patients group (41.2\%) and doctor group (53\%) (P= $0.005)$. Moreover, regarding the maximum chance of tissue rejection that was acceptable, average values of each group's re- 


\section{Fig. 7. Expectations in laryngeal allotransplantation}

The expectations (general public, kidney transplant recipients, post-laryngectomy patients, and doctors) of quality of life and the functional and aesthetic outcomes in laryngeal allotransplantation.

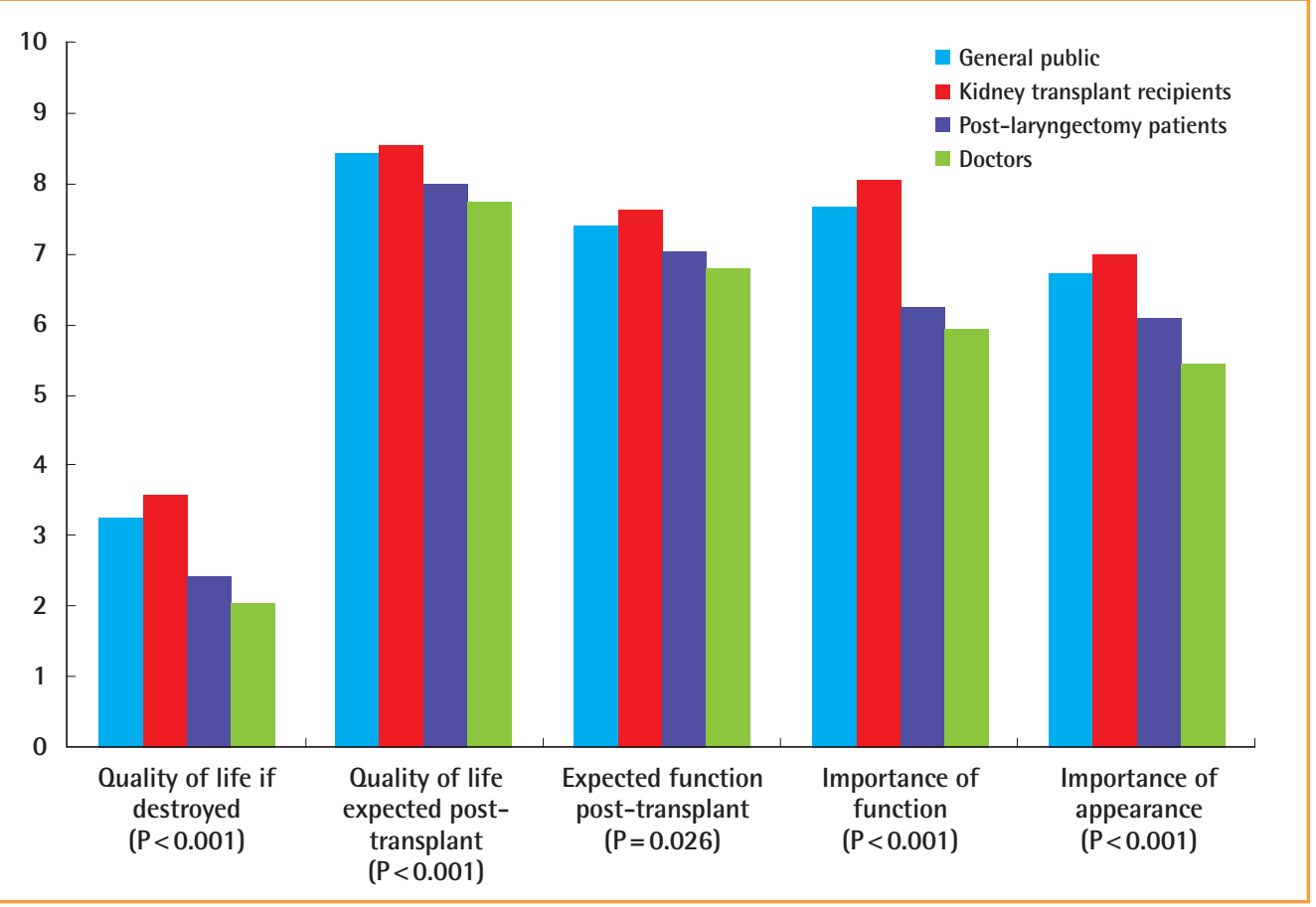

$(\mathrm{P}=0.026)($ Fig. 7).

Importance of function and appearance

It has shown that the amelioration of laryngeal appearance after LA matters to the general public (7.66), kidney transplant recipients (8.06), post-laryngectomy patients (6.24) and doctor (5.94) groups $(\mathrm{P}<0.001)$. For whether appearance is more important than having a normal voice, each four subjects groups responded as the general public (6.72), kidney transplant recipients (6.98), post-laryngectomy patients (6.09) and doctor (5.44) groups $(\mathrm{P}<0.001)$ (Fig. 7).

\section{DISCUSSION}

This study has examined the risk acceptance and expectation of LA based on survey results for the four groups: general public, kidney transplant recipients, post-laryngectomy patients, and doctors. All four groups responded differently at various levels of their perception in risk acceptance and expectations. The kidney transplant recipients reported the highest risk acceptance and expectations, and the doctor group the lowest. Comparison among the four groups was statistically significant, but there was no consistency in all items and there were statistically insignificant differences in post-hoc multiple comparisons occasionally.

The results derived from this study are similar to those of existing overseas publications [15-18], and the research conducted by Park et al. [19] and Lee et al. [20]. In their studies, the risk 
acceptance and expectations of each subject group were of the same order as this study, regardless of the type of organ transplanted, including foot, kidney, hand, both hands, larynx, partial face, and full face. Similar with the Reynolds et al. [18]'s research about the comparison among controls, kidney transplant recipients, and post-laryngectomy patients, this study has shown that kidney transplant recipients acknowledge the highest risk acceptance and expectation. Also, studies of facial allotransplantation by Park et al. [19] and of hand and foot allotransplantation by Lee et al. [20] which both conducted comparative studies between general public, medical students and doctors, had similar results to those of our study of LA, with the general public having the highest and the doctors having the lowest acceptance and expectations.

Barker et al. [17] suggested that the observed differences between the groups can be explained because "the different frames of reference led the different groups to evaluate the risks and benefits differently." The term "frames of reference" refers to the criteria of particular principles or rational thought when evaluating a phenomenon, a behavior, norms, and values. The various risk acceptance and expectations in this study about LA were indicated by the different frames of reference for each group. Tolerable risk acceptance and expectation of the kidney transplant recipients are relatively high in this survey. It can be interpreted as they had had an extensive understanding of the immunosuppressive therapy through their previous experiences and with those experiences they are willing to enhance their own threshold of acceptable risk approval. Likewise, general public have also shown the comparatively high risk acceptance and expectation during the survey. It can be assessed as their answers were more influenced by the anticipation of a successful result rather than socio-cultural background or the fear of surgery. Kidney transplant recipients and general public illustrate high levels of risk acceptance and expectation, whereas post-laryngectomy patients do not. It is possible for post-laryngectomy patients who are soon receiving the larynx, to be frightened about numerous side effects of immunosuppression, histoincompatibility and additional procedures. For the doctors with the lowest risk acceptance and expectations, they seemed to be more aware of the limitations of a gap between expectations and results compared with other groups and they were concerned about the side effects of immunosuppression and tissue rejection based on their own clinical experience and medical knowledge.

The limitation of this study was that the targeted sample and regions were confined. In the case of the general group who were guardians of out- and in-patients at the hospital, they may have been prejudiced to the survey depending on the type and severity of their dependent patients' illnesses. Furthermore, the doctors who participated in the survey had their own medical knowledge and clinical experience, but there are certain limitations to generalize these findings, as clinical experiences are bound by each participant's own individual experiences. Therefore, in order to perform a more meaningful and effective study, we suggest extending the size and scope of the sample population to a more diverse age group and a greater variety of occupations, especially for recruiting members of the general public group. Moreover, if the doctors were able to classify the degree of their own clinical experience with side effects of immunosuppression and tissue rejection, this would benefit any research performed in risk acceptance and expectations and thus will help generalizing the findings in the future.

Thus, this survey has several critical points about the selection of the sample groups and the interpretation of its results, and it can be the cornerstone for more diverse and extensive research in the future. Along with the increased income level of patients and the focus on quality of life, patients' interest in their appearance has increased. Therefore, doctors should endeavor to meet the growing needs of patients by minimizing the risks during the procedure of LA, rather than focusing on lowered expectations and the potential side effects. For the general public, a critical attitude towards information provided by the mass media is required to avoid ambiguous anticipations and illusion of composite tissue allotransplantation. Additionally, sufficient communication between patient and doctor before surgery will allow the general public to have more realistic expectations.

In addition, more invaluable and significant outcomes will be obtained for risk acceptance and expectations if the surgery of composite tissue allotransplantation including larynx improves in the future owing to developments in immunologic technology.

This study examined the disparate perception between specific population groups of the risks and benefits of using LA for the promotion of the quality of life. All four study groups responded differently to a survey of the awareness of risk acceptance and expectations in the procedure of larynx transplantation. By addressing the information gaps about LA in the different populations that have been highlighted from this survey, we suggest that LA can become a more viable alternative to classical surgery with resultant improved quality of life for patients.

\section{REFERENCES}

1. McNeil BJ, Weichselbaum R, Pauker SG. Speech and survival: tradeoffs between quality and quantity of life in laryngeal cancer. N Engl J Med 1981;305:982-7.

2. Work WP, Boles R. Larynx: replantation in the dog. Arch Otolaryngol 1965;82:401-2. 
3. Ogura JH, Kawasaki M, Takenouchi S, et al. Replantation and transplantation of the canine larynx. Ann Otol Rhinol Laryngol 1966;75:295-312.

4. Silver CE, Rosen RG, Dardik I, et al. Transplantation of the canine larynx. Ann Surg 1970;172:142-50.

5. Kluyskens P, Ringoir S. Follow-up of a human larynx transplantation. Laryngoscope 1970;80:1244-50.

6. Strome M, Stein J, Esclamado R, et al. Laryngeal transplantation and 40-month follow-up. N Engl J Med 2001;344: 1676-9.

7. Tintinago LF, Herrera DA, Medina E, et al. Ultrasonographic evaluation of a vascularized tracheal transplantation. J Ultrasound Med 2005;24:1145-9.

8. Duque E, Duque J, Nieves M, et al. Management of larynx and trachea donors. Transplant Proc 2007;39:2076-8.

9. Lorenz RR, Hicks DM, Shields RW Jr, et al. Laryngeal nerve function after total laryngeal transplantation. Otolaryngol Head Neck Surg 2004;131:1016-8.

10. Birchall MA, Lorenz RR, Berke GS, et al. Laryngeal transplantation in 2005: a review. Am J Transplant 2006;6:20-6.

11. Khariwala SS, Lorenz RR, Strome M. Laryngeal transplantation: research, clinical experience, and future goals. Semin Plast Surg 2007;21:234-41.

12. Tobin GR, Breidenbach WC 3rd, Ildstad ST, et al. The history of human composite tissue allotransplantation. Transplant Proc 2009;41:466-71.

13. Barker JH, Vossen M, Banis JC Jr. The technical, immuno- logical and ethical feasibility of face transplantation. J Surg 2004;2:8-12.

14. Shapiro PA, Kornfeld DS. Psychiatric aspects of head and neck cancer surgery. Psychiatr Clin North Am 1987;10:87100.

15. Cunningham M, Majzoub R, Brouha PR, et al. Risk acceptance in composite tissue allotranplantation reconstructive procedures. Eur J Trauma 2004;30:12-6.

16. Brouha P, Naidu D, Cunningham M, et al. Risk acceptance in composite-tissue allotransplantation reconstructive procedures. Microsurgery 2006;26:144-9.

17. Barker JH, Furr A, Cunningham M, et al. Investigation of risk acceptance in facial transplantation. Plast Reconstr Surg 2006;118:663-70.

18. Reynolds CC, Martinez SA, Furr A, et al. Risk acceptance in laryngeal transplantation. Laryngoscope 2006;116:1770-5.

19. Park SR, Kim JH, Hwang JH, et al. Investigation of risk acceptance and expectations in facial allotransplantation. J Korean Soc Plast Reconstr Surg 2010;37:555-60.

20. Lee DH, Kim JH, Hwang JH, et al. Investigation of risk acceptance and expectations in hand and foot allotransplantations. J Korean Soc Plast Reconstr Surg 2011;38:427-37.

21. Kim HM, Kim JH, Hwang JH, et al. Evaluation of reliability and validity of the louisville instrument for transplantation (LIFT) in Korean population. J Korean Soc Plast Reconstr Surg 2011;38:245-50. 